\title{
Evaluating efficiency and robustness in cilia design
}

\author{
Hanliang Guo and Eva Kansq* \\ Department of Aerospace and Mechanical Engineering, \\ University of Southern California, Los Angeles, California 90089, USA
}

(Dated: December 20, 2015)

\begin{abstract}
Motile cilia are used by many eukaryotic cells to transport flow. Cilia-driven flows are important to many physiological functions, yet a deep understanding of the interplay between the mechanical structure of cilia and their physiological functions in healthy and diseased conditions remains elusive. For developing such understanding, one needs a quantitative framework for assessing cilia performance and robustness when subject to perturbations in the cilia apparatus. Here, we link cilia design (beating patterns) to function (flow transport) in the context of experimentally- and theoretically-derived cilia models. We particularly examine the optimality and robustness of cilia design. Optimality refers to efficiency of flow transport, while robustness is defined as low sensitivity to variations in the design parameters. We find that suboptimal designs can be more robust than optimal ones. That is, designing for the most efficient cilium does not guarantee robustness. These findings have significant implications on the understanding of cilia design in artificial and biological systems.
\end{abstract}

\section{INTRODUCTION}

Cilia are hair-like structures, typically tens of micrometers in length, that protrude from the surface of eukaryotic cells. They can be found in many aquatic and terrestrial species from the unicellular protozoan Paramecium to humans. In mammals, cilia are present on many cells in the body, either in large groups on a single cell, as in the case of motile cilia, or as solitary structures, as in the case of primary and nodal cilia [1. Motile cilia, the focus of this work, are found on the epithelial cells of the trachea [2 4], ependymal cells in the brain [5, 6], and cells lining the oviduct and epididymis of the reproductive tracts [7. They normally beat in an orchestrated fashion resulting in fluid movement and cell transport 88. Great advances have been made in demonstrating the importance of ciliary transport to many physiological functions [9, 10, and in unraveling the underlying fluid-structure interactions at the cilia scale [11-15. However, a deep understanding of the interplay between the mechanical structure of cilia and their physiological functions and how ciliary dysfunction can lead to severe disease and developmental pathologies remains elusive.

It is therefore important to apply quantitative measures that link cilia mechanics (e.g., cilia beating patterns) to function (e.g., flow transport) in both healthy and diseased states [16, 17]. The lack of such standardized measures is in part due to the use of disparate research approaches in the biological and physical sciences. In health-related research, a traditional approach is to use in vitro ciliated cell cultures, from which the fundamental structure-function relationships are inferred. However, the clinical use of such in vitro cell cultures has been mostly qualitative. In biofluid mechanics research, an increasingly popular approach consists of computing

\footnotetext{
* kanso@usc.edu
}

the ideal kinematics for a single function, such as optimal fluid transport in cilia acting both individually 18 and collectively [19]. This optimization approach requires a sophisticated mathematical and computational apparatus to arrive at the optimal cilia kinematics. The optimal result provides valuable insights into ciliary design, but fails to explain, let alone evaluate, the variation in cilia kinematics and how deviations from such kinematics affect cilia function. Here, we posit that robustness under perturbations to cilia kinematics, whether due to environmental or structural causes, is of paramount importance for healthy cilia function. As such, lack of robustness can be linked to cilia dysfunction and disease. We therefore present an alternative approach that emphasizes the design principles amenable to a given function (flow transport), and the robustness of such function under variations in the cilia design parameters.

The beating cycle of a cilium typically consists of two phases: an effective stroke aimed at generating flow followed by a recovery stroke during which the cilium returns to its initial position. During the effective stroke, the cilium moves in an almost straight configuration in a plane normal to the cell surface, while in the recovery stroke, it bends parallel to the cell surface while exhibiting large curvatures and possibly moving out of the normal plane. The details of the cilia beat kinematics depend on the cell type, but the exact physical and/or biological mechanisms that select or constrain these kinematics are not well understood. We posit that optimality is not the main mechanism driving this selection. This conviction is in part based on a recent comparative study of the performance of two cilia beating patterns taken from two experimental systems, namely, cilia from a swimming microorganism and rabbit tracheal cilia. In the first system, cilia are used for swimming while in the second, they are used for fluid transport. If cilia performance were hydrodynamically optimal, one would expect the transport-specific cilia to outperform the swimmingspecific cilia in fluid transport, and vice versa. However, 
by comparing the two types of cilia, we found the cilia beating pattern taken from the swimming system to be consistently superior to the other in three different hydrodynamic performance metrics. These findings imply that cilia beating kinematics need not be optimal hydrodynamically [20]. Further, in mammalian cells, cilia beating motion takes a minimal fraction of the metabolic budget of the body. It is therefore unlikely, from the perspective of evolutionary biology, that the energetic cost associated with the beating of a cilium has posed a major selective pressure on its beat kinematics. In contrast, the energetic cost associated with locomotion consumes a significant portion of the metabolic budget in almost all motile organisms [21], thus justifying the need for an optimal or quasi-optimal gait. This is also true in cilia-driven locomotion such as in the protozoan Paramecium 222. The Paramecium uses more than half its total energy consumption for swimming, while its hydrodynamic swimming efficiency is estimated to be as low as $0.77 \%$. But it is important to distinguish between optimizing the cilium kinematics and optimizing the swimming gait. The latter involves, in addition to the cilium kinematics, the coordination between multiple cilia and different cilia types as well as their distribution on the underlying surface and the geometric properties of that surface.

In this study, we examine the beating kinematics of individual cilia in relation to their function in fluid transport. We propose three reduced design parameters that capture the salient kinematic features of motile cilia, namely, the leaning angle in the direction of the effective stroke, the beating amplitude of the effective stroke, and the out-of-plane angle. We present a straightforward approach for extracting these design parameters from any cilia beating pattern, including those obtained experimentally from high-speed image sequences. We then present a mathematical family of cilia-like kinematics which can be constructed for any combination of design parameters.

We validate this choice of "generic" cilia-like kinematics by comparing it to experimentally-derived cilia kinematics before we use it to investigate questions of optimality and robustness of cilia design. Optimality here is defined in terms of the efficiency of flow transport, while robustness refers to lack of sensitivity in transport efficiency to variations in the design parameters. We find that suboptimal kinematics are more robust than optimal kinematics. That is, there is an interplay between optimality and robustness in cilia design. To our knowledge, our study is the first to propose robustness as an important criterion in cilia design. Our findings provide a quantitative framework for investigating cilia performance under various operating conditions and, thus, have significant implications on the understanding of cilia design in artificial and biological systems.

\section{MODEL AND METHOD}

We consider a cilium whose base point is chosen to coincide with the origin of properly-chosen Cartesian coordinates $(x, y, z)$. The length of the cilium is $l$. The cilium beats periodically in the half infinite domain $y>0$ with frequency $\omega$ and period $T=2 \pi / \omega$.

The beating motion of the cilium is represented by $\mathbf{x}_{c}(s, t) \equiv\left(x_{c}(s, t), y_{c}(s, t), z_{c}(s, t)\right)$, where $s$ is the arclength along the cilium's centerline from its base $(0<$ $s<l)$ and $t$ is time $(0<t<T)$. To describe the cilia beating kinematics from experimental observation of cilia beat patterns [2, 23], we write each component of $\mathbf{x}_{c}(s, t)$ using a Taylor series expansions in $s$ and Fourier series expansion in $t$. We then calculate the series coefficients that best fit the experimental data [2, 23] subject to the constraint that the total length of the cilium is conserved, see Fig. 1. Here, for consistency and without loss of generality, we consider the effective stroke direction to be pointing in the positive $x$-direction. It is clear from Fig. 1 that the details of the cilia beating kinematics vary depending on the cell type, but qualitatively they all follow the same trend. Further, all these examples correspond to planar cilia kinematics. Based on the original planar images in Sleigh [23], and Fulford \& Blake [2], it is relatively straightforward to assess whether the bending kinematics is planar by comparing the projected cilium length in the experimental image sequence of the beat cycle to absolute cilium length [23]. In each of the cases presented here, we found little change in the cilium's overall length over one beating cycle (with standard deviation about $3 \%$ ), indicating that the cilium bending motion is mostly two-dimensional. Note that in the cases when the cilia kinematics is non-planar, it is very difficult to uniquely reconstruct the out-of-plane motion from planar images. For this reason, we only reconstructed planar kinematics in Fig. 1 .

In order to facilitate the comparison between the beating kinematics of various cilia type, it is useful to describe the cilia beating patterns mathematically using a small number of parameters. Concise mathematical descriptions date back to G. I. Taylor's swimming sheet [24], albeit for modeling symmetric beating motion. Asymmetric beating patterns were later described using various approaches, including the "biased baseline" mechanisms [25, the Taylor and Fourier series expansions [2] employed above, and more recently, efficient polynomial expansions [26] and curvature-based Fourier series expansions [27]. These methods can reconstruct the beating patterns from given experimental data, but often require sophisticated machinery and large number of parameters. Here, we introduce three reduced "cilia design" parameters that can be used to reconstruct any beating patterns in 3D: the leaning angle $\lambda_{o}$, the beating amplitude $\alpha_{o}$, and the swinging angle $\beta_{o}$, as shown in Fig. 2. To define these parameters, we first let $\lambda$ be the angle between the straight line pointing from the cilium base to the cilium tip and the $(y, z)$ plane and $\beta$ be the angle between the 


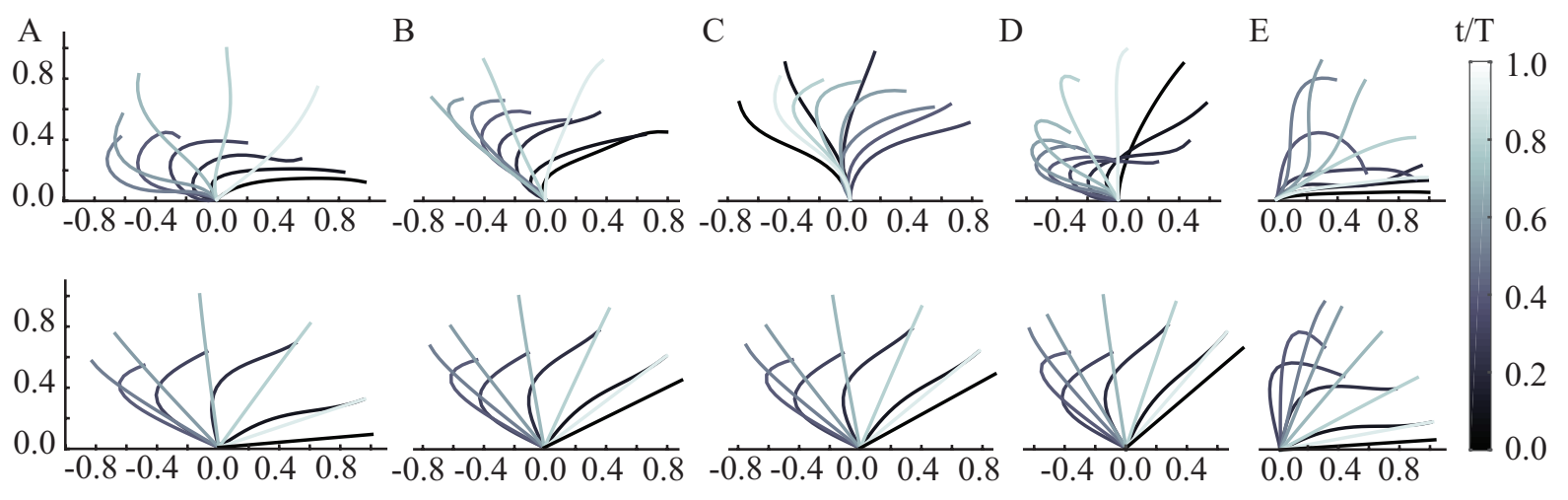

FIG. 1: Cilia beating patterns reconstructed from: (top row) experimental data extracted from Sleigh [23, and Fulford \& Blake [2]. A: Didinium; B: Paramecium; C: Rabbit tracheal cilia; D: Sabellaria gill; E: Opalina; (bottom row) mathematical model presented in this paper where the values of $\left(\lambda_{o}, \alpha_{o}\right)$ are extracted from the experimental images in the top row.
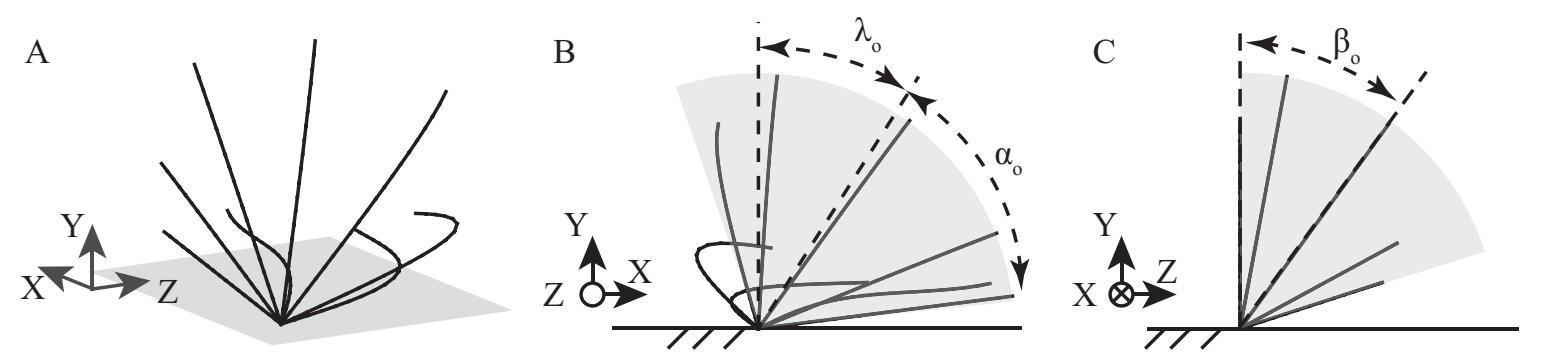

FIG. 2: Reduced cilia design parameters $\lambda_{o}, \alpha_{o}$ and $\beta_{o}$. A: three-dimensional beating pattern of the cilium. B: projection onto the plane of the effective stroke showing the leaning angle $\lambda_{o}$ and beating amplitude $\alpha_{o}$. C: projection onto the plane normal to the effective stroke showing the out-of-plane swinging angle $\beta_{o}$.

plane containing the curved cilium and the $(x, y)$ plane. Positive $\lambda$ are taken to be counter-clockwise about the positive $z$-axis, whereas positive $\beta$ are measured clockwise about the positive $x$-axis. Basically, $\lambda$ describes the amount by which the cilium is leaning in the direction of the effective stroke, while $\beta$ describes the amount by which the cilium leans away the plane of the effective stroke in the positive $z$-axis. The three design parameters $\lambda_{o}, \alpha_{o}$ and $\beta_{o}$ can then be defined as follows:

$$
\begin{aligned}
\lambda_{o} & =\frac{1}{2}\left[\max _{0 \leq t<T}(\lambda)+\min _{0 \leq t<T}(\lambda)\right], \\
\alpha_{o} & =\frac{1}{2}\left[\max _{0 \leq t<T}(\lambda)-\min _{0 \leq t<T}(\lambda)\right], \\
\beta_{o} & =\frac{1}{2}\left[\max _{0 \leq t<T}(\beta)+\min _{0 \leq t<T}(\beta)\right] .
\end{aligned}
$$

For the examples reported in Fig. 1, $\beta$ and $\beta_{o}$ are identically zero. These three parameters capture the salient features of the actual beating patterns but not all their details. The main reason we choose these parameters is that they are easy to access from experimental images and they are amenable to a low-order mathematical representation of asymmetric cilia beating patterns as discussed next.
We now introduce a mathematical family of functions that emulates cilia beating kinematics. This mathematical family can be viewed as a "blue print" for generating cilia beating kinematics that satisfy given cilia design parameters $\left(\lambda_{o}, \alpha_{o}, \beta_{o}\right)$. Basically, we use a sinusoisal function to describe the shape of the cilium at different times in local Cartesian coordinates; we then rotate the shape according to the design parameters $\lambda_{o}, \alpha_{o}$ and $\beta_{o}$ to generate cilia-like kinematics. To this end, we let

$$
\zeta(\xi, t)=l A(t) \sin \left(\frac{3 \pi}{2} \frac{\xi}{l}\right), \quad A(t)=1-\cos \left(\pi \frac{t}{T}\right) .
$$

Here, $(\xi, \zeta)$ are local Cartesian coordinates and must satisfy the constraint that the total length of the cilium is constant for all time $t$. Namely, one has $\mathrm{d} s=\sqrt{(\mathrm{d} \xi)^{2}+(\mathrm{d} \zeta)^{2}}$, which yields $\int_{0}^{l} \mathrm{~d} s=$ $\int_{0}^{l} \mathrm{~d} \xi \sqrt{1+(\mathrm{d} \zeta / \mathrm{d} \xi)^{2}}=l$. By virtue of the implicit function theorem, one can use this equality together with (2) to write $\xi(s, t)$ and $\zeta(s, t)$ as functions of arc-length and time. Fig. $3 \mathrm{~A}$ is a depiction of the kinematics obtained from these generating functions. The wave number $3 \pi / 2$ is chosen so that the cilium does not exhibit both positive and negative curvatures at any given time, consistent with experimental observation of cilia beating patterns 

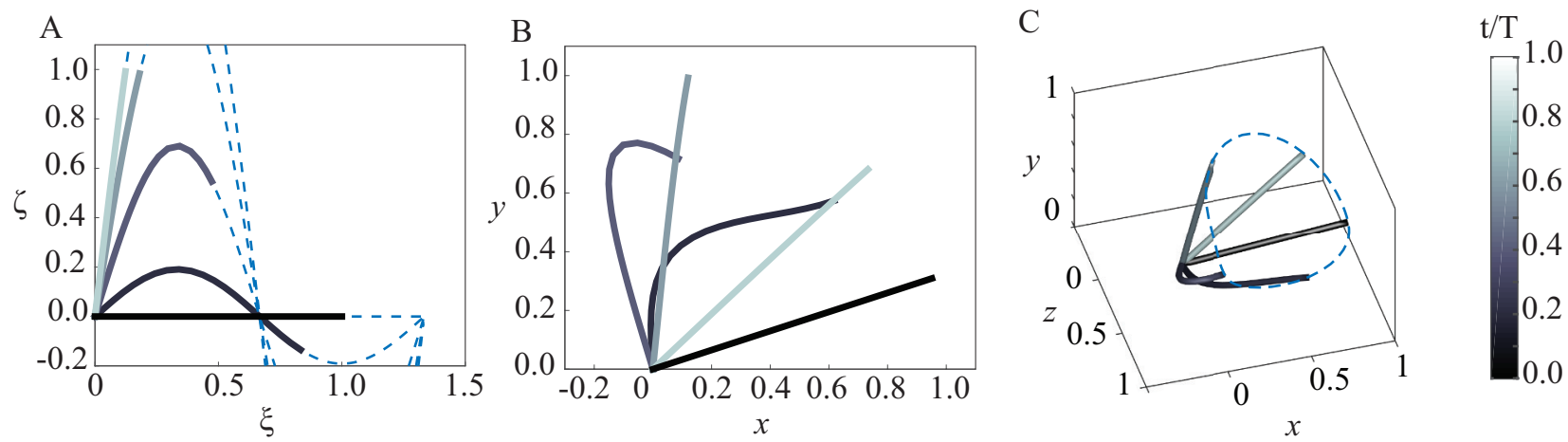

FIG. 3: Generic cilia-like kinematics. A: shapes of the strokes at select times are constructed from truncated sinusoidal functions that ensure conservation of total length of the cilium. B: planar beating patterns at select times. C: beating patterns at selected phases in three-dimensional space. Parameter values in all subfigures are $\lambda_{o}=.2 \pi, \alpha_{o}=.2 \pi, \beta_{o}=.2 \pi$

(see Fig. 1 and analysis in [23]). The time-dependent wave amplitude $A(t)$ is chosen so that the cilium is less curved in the effective stroke $(0.5 T<t<T)$ than in the recovery stroke $(0<t<0.5 T)$. Note that one can expand this representation in terms of a $2^{\text {nd }}$ order Taylor expansion in $s$, given that no inflection points in the cilium shape are allowed, and a Fourier series expansions in time. The coefficients of these expansions are omitted here for brevity.

Cilia-like kinematics with desired values of $\left(\lambda_{o}, \alpha_{o}, \beta_{o}\right)$ can be constructed using the "blue print" $(\xi(s, t), \zeta(s, t))$ as follows. We first write the instantanous leaning angle $\lambda(t)$ and swinging angle $\beta(t)$ as explicit functions of time

$$
\begin{aligned}
& \lambda(t)=\lambda_{o}+\alpha_{o} \cos \left(2 \pi \frac{t}{T}\right), \\
& \beta(t)=\beta_{o}\left[1+\sin \left(2 \pi \frac{t}{T}\right)\right] .
\end{aligned}
$$

We then rotate $(\xi, \zeta)$ about the $z$-axis by an angle $\lambda-\arctan (\xi(l, t) / \zeta(l, t))$ to produce the cilium bending motion in the $(x, y)$ plane, see Fig. $3 \mathrm{~B}$. To make the cilium swing out of the vertical plane, we perform a second rotation about the $x$-axis by the angle $\beta$, see Fig. $3 \mathrm{C}$. At the end of these two rotations, one obtains cilia-like kinematics $\left(x_{c}(s, t), y_{c}(s, t), z_{c}(s, t)\right)$ that satisfy the desired design parameters $\left(\lambda_{o}, \alpha_{o}, \beta_{o}\right)$. This family of beating kinematics qualitatively resembles the beating patterns of biological cilia in that the effective stroke is straight and perpendicular to the base surface while the recovery stroke is curly and close to the base surface. The beating kinematics, by construction, spend equal amount of time in effective and recovery strokes. The hydrodynamic performance, however, is independent of time because the flow in Stokes regime is essentially a geometric problem.

We use $l$ and $T$ to scale length and time, respectively. All variables are thereafter non-dimensional. The fluid motion is governed by the non-dimensional Stokes equation and incompressibility condition at zero Reynolds number,

$$
-\nabla p+\mu \nabla^{2} \mathbf{u}=\mathbf{0}, \quad \nabla \cdot \mathbf{u}=0,
$$

where $p$ is the pressure field, $\mu$ is the dimensionless viscosity, and $\mathbf{u}$ is the fluid velocity field. The boundary condition at the cilium and base surface is a no-slip condition

$$
\left.\mathbf{u}\right|_{\text {boundary }}=\left\{\begin{array}{ll}
\mathbf{u}_{c} & \text { at the cilium } \\
\mathbf{0} & \text { at the base surface }
\end{array},\right.
$$

where $\mathbf{u}_{c}$ is the prescribed velocity of the cilium, based on the kinematics reconstructed either from experimental images or the mathematical formulation.

We solve (4) and (5) numerically using the regularized Stokeslet method [28]. The cilium is approximated by a distribution of regularized Stokeslets along its centerline, together with an "image" distribution to impose the noslip boundary conditions at the base surface [29]. The velocity at any point $\mathbf{x}$ induced by a regularized Stokeslet of strength $\mathbf{f}_{i}$ located at $\mathbf{x}_{i}$ and its images can be written as $\mathbf{G}_{s}\left(\mathbf{x}-\mathbf{x}_{i}\right) \cdot \mathbf{f}_{i}$, where $\mathbf{G}_{s}$ is the regularized Green's tensor-valued function given by Ainley et al. [29]. The total velocity generated by all regularized Stokeslets is then

$$
\mathbf{u}(\mathbf{x})=\sum_{i=1}^{n} \mathbf{G}_{s}\left(\mathbf{x}-\mathbf{x}_{i}\right) \cdot \mathbf{f}_{i}
$$

where $n$ is the total number of regularized Stokeslets. The expression in (6) is substituted into (5) to obtain a system of equations that can be used to compute the strengths $\mathbf{f}_{i}$ of the regularized Stokeslets. Once $\mathbf{f}_{i}$ are known, the flow field can be reconstructed everywhere.

Let $Q_{x}$ and $Q_{z}$ denote the flow transported by the cilium in the $x$ - and $z$-directions, respectively. One has (see [15, 30, 31] for more details)

$$
Q_{x}=\frac{1}{\mu \pi} \int_{0}^{l}\left(\mathbf{f}_{i} \cdot \mathbf{e}_{x}\right) y d s, \quad Q_{z}=\frac{1}{\mu \pi} \int_{0}^{l}\left(\mathbf{f}_{i} \cdot \mathbf{e}_{z}\right) y d s
$$


where $\mathbf{e}_{x}$ and $\mathbf{e}_{z}$ are the unit vectors in $x$ - and $z$ directions. These expressions are based on the fact that the net flux generated by a Stokeslet over an infinite plane is directly proportional to the height of the Stokeslet above the plane [30]. Now, consider the time average of the flow rates per cycle: $\left\langle Q_{x}\right\rangle=\frac{1}{T} \int_{0}^{T} Q_{x} d t$ and $\left\langle Q_{z}\right\rangle=\frac{1}{T} \int_{0}^{T} Q_{z} d t$. The total average flow rate is $\langle Q\rangle=\sqrt{\left\langle Q_{x}\right\rangle^{2}+\left\langle Q_{z}\right\rangle^{2}}$.

To compute the internal power spent by the cilium during the beating cycle, we consider each cilium to be an inextensible elastic filament and adopt the Kirchhoff equations of motion for a rod [18, 20]

$$
\frac{\partial \mathbf{N}}{\partial s}-\mathbf{f}=0, \quad \frac{\partial \mathbf{M}}{\partial s}+\mathbf{t} \times \mathbf{N}+\mathbf{q}=0 .
$$

Here $\mathbf{N}(s, t)$ and $\mathbf{M}(s, t)$ are the internal tension and elastic moment respectively, $\mathbf{f}(s, t)$ is the force exerted by the cilium on the surrounding fluid, $\mathbf{t}=\partial \mathbf{x}_{c} / \partial s$ is the unit tangent to the cilium, and $\mathbf{q}(s, t)$ is the internally generated moment per unit length. The internal bending moment $\mathbf{q}$ resembles the moments generated by the cilium internal motors.

The force distribution $\mathbf{f}$ is computed by dividing the local Stokeslet strength $\mathbf{f}_{i}$ by the distance between neighboring Stokeslets along the cilium. We assume a linear constitutive relation between the elastic moment $\mathbf{M}$ along the cilium and the deformation [18, 20. Namely, we let $\mathbf{M}=B \mathbf{D}$, where $B$ is the bending rigidity and $\mathbf{D}=\mathbf{t} \times(\partial \mathbf{t} / \partial s)$ is the Darboux vector. Substituting $\mathbf{M}=B \mathbf{D}$ into $(8)$, one gets the expression of the internal moments generated along the cilium

$$
\mathbf{q}=B \frac{\partial^{2} \mathbf{t}}{\partial s^{2}} \times \mathbf{t}+\mathbf{t} \times \int_{s}^{l} \mathbf{f}(\tilde{s}, t) \mathrm{d} \tilde{s} .
$$

The average power $\langle P\rangle$ expended internally by the cilium to transport is equal to the power consumed by the internal moments q,

$$
\langle P\rangle=\left\langle\int_{0}^{l} \max (0, \mathbf{q} \cdot \boldsymbol{\Omega}) \mathrm{d} s\right\rangle,
$$

where $\boldsymbol{\Omega}=\|\dot{\mathbf{t}}(s)\| \frac{\mathbf{t} \times \dot{\mathbf{t}}}{\|\mathbf{t} \times \dot{\mathbf{t}}\|}$ is the angular velocity vector, with () denoting the time derivative. Here, only the positive works are accounted for, i.e., the cilium does not harvest energy from the environment [18, 32. This implies that the mean power spent by the cilium's internal moments is larger than the power given to the fluid. Finally, we define a dimensionless transport efficiency,

$$
\eta=\mu l^{-3} \frac{\langle Q\rangle^{2}}{\langle P\rangle},
$$

which is consistent with that employed by Osterman \& Vilfan [19] and Eloy \& Lauga [18].

\section{RESULTS}

Our goal is to evaluate the hydrodynamic performance of the beating kinematics of individual cilia as a function of the three design parameters: the leaning angle $\lambda_{o}$, the beating amplitude $\alpha_{o}$ and the out-of-plane swinging angle $\beta_{o}$. We use two evaluation metrics: the average flow rate $\langle Q\rangle$ and the efficiency $\eta$.

We discretize the cilium uniformly using 20 regularized Stokeslets. The regularization parameter is chosen to be 0.05 , which yields a cilium length-to-radius ratio about 20. Each beating cycle is discretized into 100 time steps. By way of validation, we are able to reproduce the flow rates generated by embryonic primary cilia given by Smith et al. 31] with these discretization parameters.

We begin by considering planar cilia beat kinematics, for which $\beta_{o}=0$. We examine the performance of the cilium as a function of $\lambda_{o}$ and $\alpha_{o}$. Namely, we vary $\lambda_{o}$ in the range from $-0.15 \pi$ to $0.45 \pi$ and we vary $\alpha_{o}$ from 0 to $0.45 \pi$ using a step size of $0.01 \pi$. Combinations of parameters for which $\lambda_{o}+\alpha_{o}>0.5 \pi$ lead to prescribed cilium motion that penetrate the base surtface. These combinations are considered unrealistic and not considered. Note that accurate information regarding the angular frequency and bending rigidity of cilia is sparse. Here, as well as in the rest of this section, we use the information available for the Paramecium as a proxy to obtain the right order of magnitude for the cilia angular frequency and bending rigidity. The typical length of the Paramecium cilia is about $10 \mu \mathrm{m}$, typical angular frequency is about $200 \mathrm{rad} \cdot \mathrm{s}^{-1}$, and the bending rigidity is estimated to be $B=25 \mathrm{pN} \cdot \mu \mathrm{m}^{2}$ (see [18, 33, 34]). We thus use the characteristic length $l_{c}=10 \mu \mathrm{m}$, time $T_{c}=2 \pi / 200=0.0314 \mathrm{~s}$, viscosity $\mu_{c}=10^{-3} \mathrm{~Pa} \cdot \mathrm{s}$. The non-dimensional bending rigidity is then given by $B /\left(\mu_{c} l_{c}^{4} T_{c}^{-1}\right)=0.0785$. Note that more recent estimates of the bending rigidity predict higher values of $B$, e.g., 35. However, the exact $B$ value does not affect the main findings of this work, which focuses mainly on a comparative analysis of cilia performance under various design parameters.

The average flow rate $\langle Q\rangle$ and transport efficiency $\eta$ for the admissible parameter values $\left(\lambda_{o}, \alpha_{o}\right)$ are shown in Fig. 4. It is clear from Fig. $4 \mathrm{~A}$ that larger beating amplitude $\alpha_{o}$ lead to larger average flow rate. Meanwhile the leaning angle $\lambda_{o}$ has a small effect on the average flow rate. The beating patterns with small beating amplitude $\left(\alpha_{o} \approx 0\right)$ correspond to traveling waves somewhat similar to those observed in the flagella of sperm cells 33, 36. However, unlike the traveling waves in natural sperm cells, these waves are constrained such that, the head and tip of the wave always lie at a constant direction from the substrate and the curvature of the wave has the same sign. If one were to use a flagellated sperm cell and adhere its head to a substrate such that its flagellum beats in such constraint traveling wave fashion, it would produce little flow.

The effect of $\alpha_{o}$ and $\lambda_{o}$ on the transport efficiency $\eta$ is 

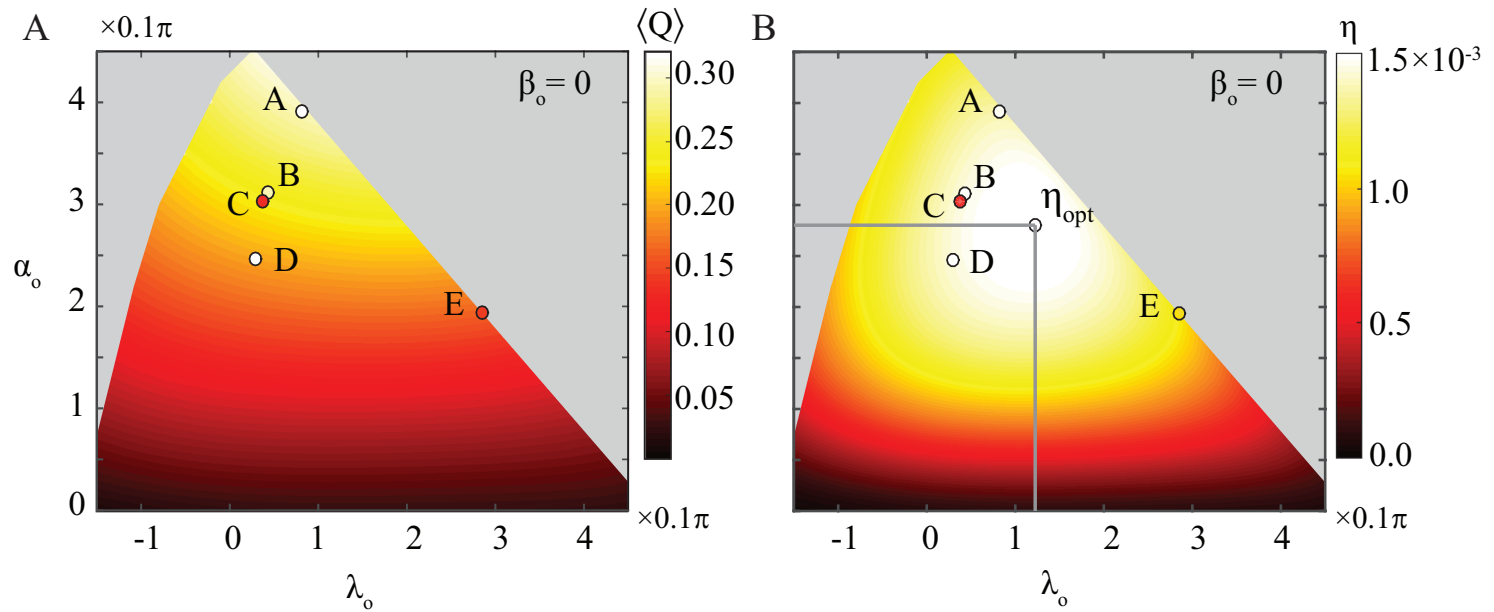

FIG. 4: Flow rate $\langle Q\rangle$ and efficiency $\eta$ generated by the generic cilia model with $\beta_{o}=0$. Flow rates and the efficiencies of the cilia beating patterns reconstructed in Fig. 1 are plotted in circles at corresponding values of $\lambda_{o}$ and $\alpha_{o}$. The color of the circle indicates the performance of these cilia using the colormap of each subfigure. Deviations between the background color and the circle color indicate deviations in the performance of the generic model and actual cilia.

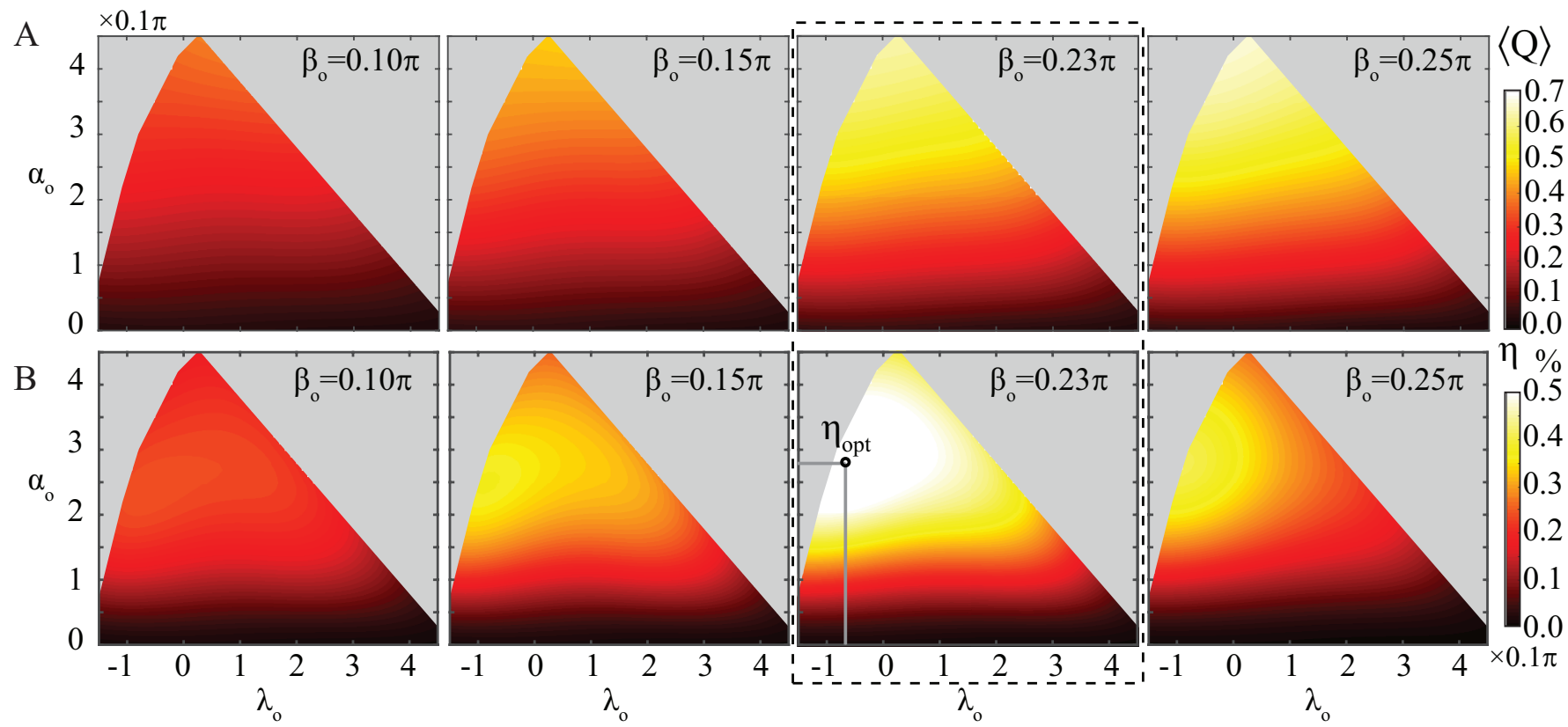

FIG. 5: A: average flow rates $\langle Q\rangle$ of generic cilia kinematica with different beating amplitude $\alpha_{o}$, leaning angle $\lambda_{o}$, and swinging angle $\beta_{o}$. B: Transport efficiencies.

depicted in Fig. 4B. The beating pattern with $\lambda_{o}=0.12 \pi$ and $\alpha_{o}=0.28 \pi$ yields the highest transport efficiency $0.15 \%$. Unlike its effect on $\langle Q\rangle$, a larger beating amplitude does not yield higher efficiency. This is because at higher $\alpha_{o}$ the cilium will need to spend more power in its motion close to the base surface to overcome the zero velocity at that surface. The extra power needed to complete such beating cycles lowers the transport efficiency.

By way of validation of these generic cilia-like kinematics, we compute the performance of the five beating patterns reconstructed from experimental data shown in Fig. 1. The leaning angles and beating amplitudes of these cilia are calculated according to (1). The flow rates and the transport efficiencies are then computed and the results are superimposed onto Fig. 4 as colored circles in accordance with their leaning angles and beating amplitudes. Clearly, the experimentally-derived beating patterns are scattered in the high efficiency zone predicted by the generic model. Furthermore, the flow rates and the transport efficiencies predicted by the generic kinemat- 
ics are close to those obtained from experimental data, except for one data point which differs from the generic model by a factor of about 2 . These findings justify our choice of both the reduced design parameters and the generic model.

We now consider three-dimensional cilia kinematics and assess the effects of the out-of-plane swinging angle $\beta_{o}$ on $\langle Q\rangle$ and $\eta$. Namely, we vary $\beta_{o}$ from zero (results in Fig. 40 to $0.25 \pi$ using increments of $0.01 \pi$. In Figs. 5 . the values of $\langle Q\rangle$ and $\eta$ are depicted for select values of $\beta_{o}$. Fig. 5A shows that a larger swinging angle $\beta_{o}$ leads to a larger average flow rate. This is similar to the effect of the beating amplitude $\alpha_{o}$ on the flow rate: the largest values of $\alpha_{o}$ and $\beta_{o}\left(\alpha_{o}=0.45 \pi, \beta_{o}=0.25 \pi\right)$ yields the largest average flow rate. The efficiency, on the other hand, is not a monotonically increasing function of $\beta_{o}$. Fig. $5 \mathrm{~B}$ shows that the efficiencies for $\beta_{o}=0.23 \pi$ are higher than those for $\beta_{o}=0.25 \pi$ at the same values of $\lambda_{o}$ and $\alpha_{o}$. In fact, we chose to depict the panels for $\beta_{o}=0.23$ because it is the out-of-plane angle that leads to maximum transport efficiency for $\lambda_{o}=\lambda_{\text {opt }}=-0.07 \pi$, $\alpha_{o}=\alpha_{\mathrm{opt}}=0.28 \pi$. The maximum efficiency value is $0.56 \%$.

To study the sensitivity of the cilia performance with respect to the design parameters $\left(\lambda_{o}, \alpha_{o}, \beta_{o}\right)$, we perturb each one of these parameters away from the optimal or most efficient combination while keeping the other two parameters the same. In particular, we let $\lambda_{o}=\lambda_{\text {opt }}+\Delta$, $\alpha_{o}=\alpha_{\mathrm{opt}}-\Delta$, and $\beta_{o}=\beta_{\mathrm{opt}}-\Delta$, where $\left(\lambda_{\mathrm{opt}}, \alpha_{\mathrm{opt}}, \beta_{\mathrm{opt}}\right)$ is the optimal parameter combination that leads to the most efficient cilium. The most efficient beating pattern and the beating patterns perturbed from the most efficient one are shown in Fig. 6

We introduce two measures of sensitivity: (i) a "global" sensitivity which evaluates the net change in flow transport $\left|\langle Q\rangle-\left\langle Q_{\text {opt }}\right\rangle\right| /\left\langle Q_{\text {opt }}\right\rangle$, and (ii) a "local" sensitivity which evaluates the rate of change in flow transport normalized by $\left\langle Q_{\text {opt }}\right\rangle$. Here, by rates of change in flow transport we mean $\left|\partial\langle Q\rangle / \partial \lambda_{o}\right|,\left|\partial\langle Q\rangle / \partial \alpha_{o}\right|$, and $\left|\partial\langle Q\rangle / \partial \beta_{o}\right|$. Similarly, the net change in efficiency $\left|\eta-\eta_{\text {opt }}\right| / \eta_{\text {opt }}$ and rates of change of efficiency $\left|\partial \eta / \partial \lambda_{o}\right|,\left|\partial \eta / \partial \alpha_{o}\right|$, and $\left|\partial \eta / \partial \beta_{o}\right|$ are, respectively, "global" and "local" measures of the sensitivity in cilia efficiency as the beating kinematics deviate from that of the most efficient cilium. A lower sensitivity implies more robustness to perturbations imposed on cilia design parameters, and vice versa.

In Fig. $7 \mathrm{~A}$ is a depiction of the global sensitivities of both the transport flow rate and efficiency as a function of the deviation $\Delta$ from the optimal cilium kinematics. Both the flow rate and efficiency are more sensitive to the beating amplitude $\alpha_{o}$ and out-of-plane swinging amplitude $\beta_{o}$ than to the leaning angle $\lambda_{o}$. For example, a perturbation $\Delta=0.1 \pi$ (dashed grey lines in Fig. $7 \mathrm{~A}$ ) in the swinging angle $\beta_{o}$ will reduce the flow rate by over $36 \%$, as compared to a reduction of $25 \%$ and $3 \%$ when varying the beating amplitude $\alpha_{o}$ and leaning angle $\lambda_{o}$ by the same amount, respectively. A similar trend is observed for the sensitivity in the transport efficiency $\eta$.
The local sensitivity measures, shown in Fig. 7B confirm these findings but indicate that for larger deviations from the optimal kinematics, the performance of the cilium becomes more sensitive to variations in beating amplitude $\alpha_{o}$. These results indicate that to maintain lower sensitivity at large perturbations away from the optimal beating kinematics, a better strategy is to allow deviations/variations in $\beta_{o}$ while restricting the variations in $\alpha_{o}$. In other words, ciliary defects that induce large variations in $\alpha_{o}$ lead to ineffective flow transport.

Fig. 7B also shows the sensitivities associated with a suboptimal choice of cilia design parameters (dashed lines). Namely, instead of perturbing the parameters from $\left(\lambda_{\mathrm{opt}}, \alpha_{\mathrm{opt}}, \beta_{\mathrm{opt}}\right)$, we consider the suboptimal parameters $\left(\lambda_{\mathrm{opt}}, \alpha_{\mathrm{opt}}, \beta^{*}\right)$, where $\beta^{*}=\beta_{\mathrm{opt}}-0.05 \pi$ and impose perturbations $\Delta$ away from this suboptimal choice. Our goal is to compare the sensitivity of optimal and suboptimal design: the design with lower sensitivity is more robust to perturbations. It is evident from Fig. 7B that the "local" sensitivities of the flow rate and efficiency associated with the suboptimal design are lower than those associated from the optimal design for almost all $\Delta$, indicating that a suboptimal design may lead to more robust performance when the design parameters are perturbed. Note that the only sensitivity component that is increased with this suboptimal choice of parameters is the sensitivity of transport efficiency with respect to the out-of-plane angle $\beta_{o}$ when the perturbation $\Delta$ is very small $\Delta<0.04 \pi$.

Finally, we set $\Delta=0.05 \pi$ (highlighted in light grey in Fig. 7) and we calculate the global and local sensitivities in transport efficiency $\eta$ for all admissible values of $\lambda_{o}$ and $\alpha_{o}$ for $\beta_{o}=\beta^{*}=\beta_{\mathrm{opt}}-\Delta$. In particular, we let $\left(\lambda^{*}, \alpha^{*}\right)$ denote the parameters that lead the highest transport efficiency $\eta^{*}$ for $\beta_{o}=\beta^{*}$, and we measure the global and local sensitivities relative to $\eta^{*}$. The results are depicted in Fig. 8A and B, respectively. In the latter, the local sensitivities with respect to the design parameters $\alpha_{o}, \lambda_{o}$ and $\beta_{o}$ are consolidated into one scalar function $\left(|\nabla \eta|-\left|\nabla \eta^{*}\right|\right) / \eta^{*}$, where $\nabla \eta$ is defined as $\nabla \eta=\sqrt{\left(\partial \eta / \partial \lambda_{o}\right)^{2}+\left(\partial \eta / \partial \alpha_{o}\right)^{2}+\left(\partial \eta / \partial \beta_{o}\right)^{2}}$. Such depiction serves as a tool to identify the design parameters $\alpha_{o}, \lambda_{o}$ that satisfy desired limits on sensitivity given a perturbation size, in this case $\Delta=0.05 \pi$. For example, to ensure minimum sensitivities and maximum robustness for this perturbation size, one would choose values of $\alpha_{o}$ and $\lambda_{o}$ that lie in the intersection of the regions that correspond to minimal sensitivities in Fig. $8 \mathrm{~A}$ and B.

A few comments on the interplay between efficiency and robustness are in order. At optimal efficiency, one has $\nabla \eta=0$ by definition, which implies infinite robustness. However, cilia are often subject to perturbations in the fluid environment as well as in the ciliary apparatus. But any perturbation away from the optimal beating pattern causes the cilium to loose efficiency. It is thus reasonable to assume that cilia often operate at suboptimal efficiency. The question then is, starting from subopti- 

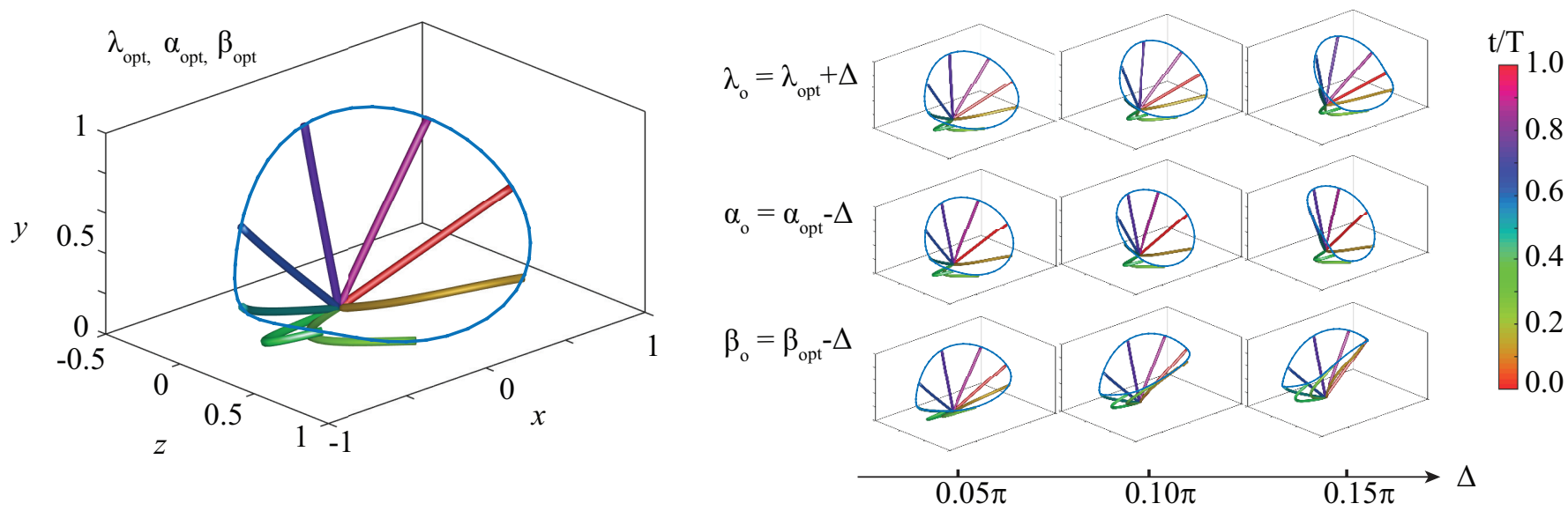

FIG. 6: Optimal (left) and suboptimal (right) beating patterns. The suboptimal beating patterns are constructed by perturbing one of the three design parameters while keeping the other two the same as in the optimal beating pattern.
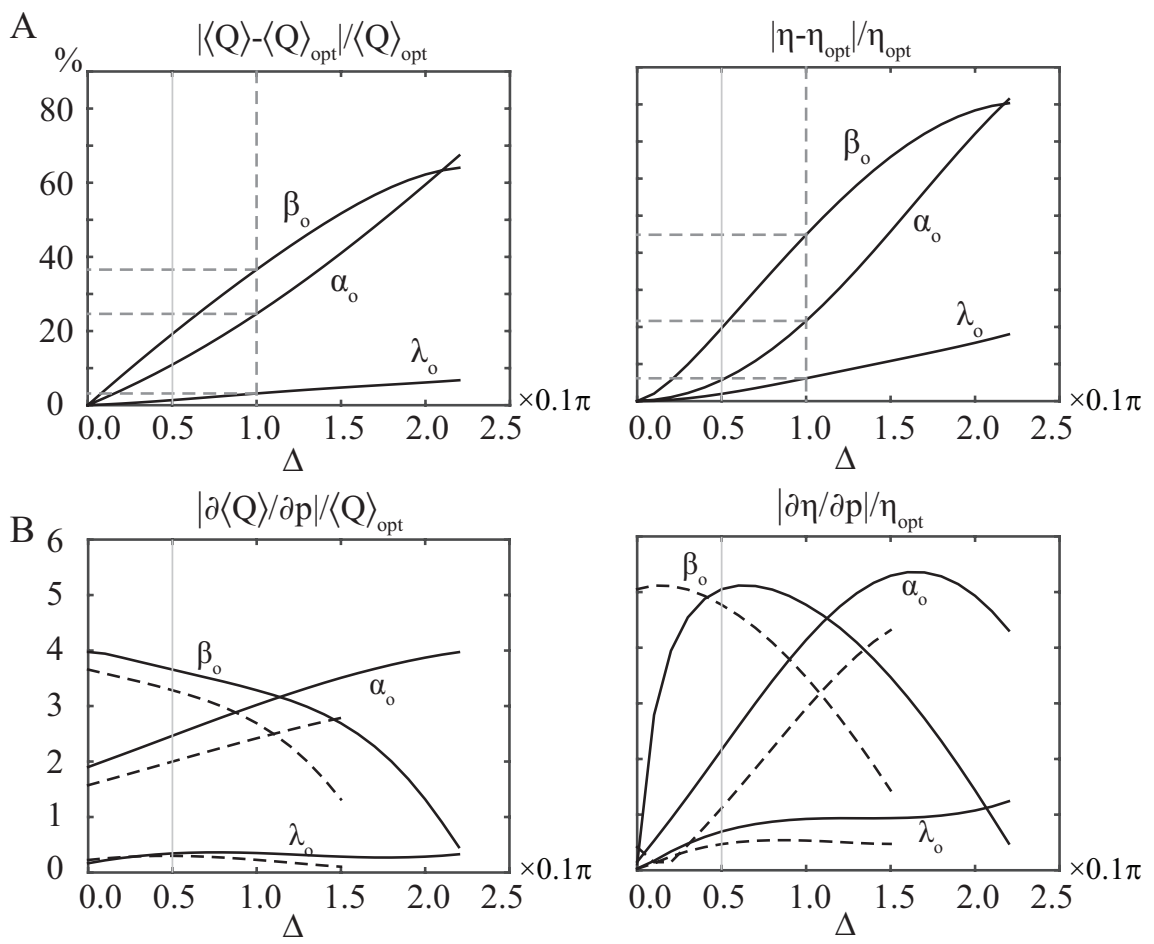

FIG. 7: Sensitivity of the net flow rate $\langle Q\rangle$ and the transport efficiency $\eta$ with respect to perturbations $\Delta$ away from the optimal design parameters $\alpha_{\text {opt }}, \lambda_{\text {opt }}$ and $\beta_{\text {opt }}$. A: "global" sensitivities or net change in $\langle Q\rangle$ and $\eta$ as a function of $\Delta$. B: "local" sensitivities or rate of change in $\langle Q\rangle$ and $\eta$ as a function of perturbations $\Delta$ away from optimal parameter values (solid lines) and away from suboptimal parameter values (dashed lines) .

mal efficiency, how is cilia transport affected by further perturbations to the cilia beating kinematics. Interestingly, we found that starting from suboptimal efficiency, cilia transport may be more robust to perturbations than starting from optimal efficiency, hence, the most efficient cilium is not the most robust.

\section{DISCUSSION}

We parameterized cilia beating kinematics using three reduced design parameters: the leaning angle $\lambda_{o}$ of the cilium in the direction of the effective stroke, the cilium beating amplitude $\alpha_{o}$, and its out-of-plane swinging angle $\beta_{o}$. We presented a straightforward method for extract- 
A

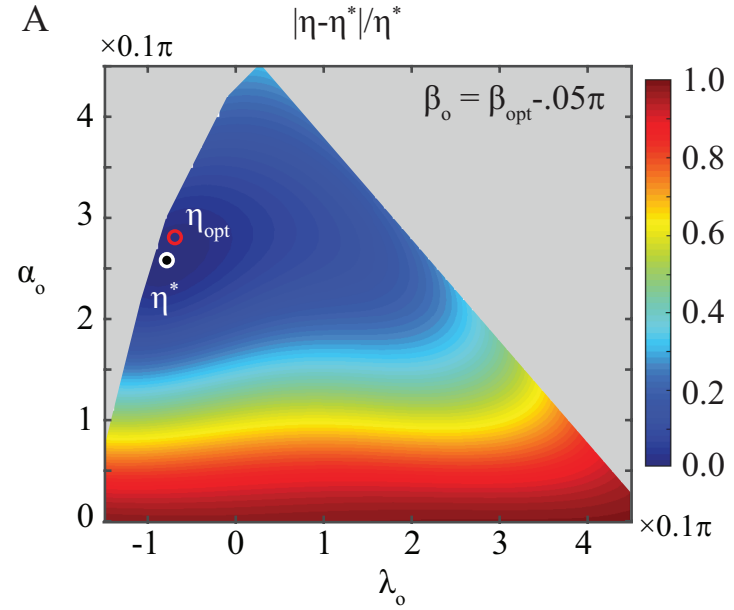

B $\quad\left(|\nabla \eta|-\left|\nabla \eta^{*}\right|\right) / \eta^{*}$

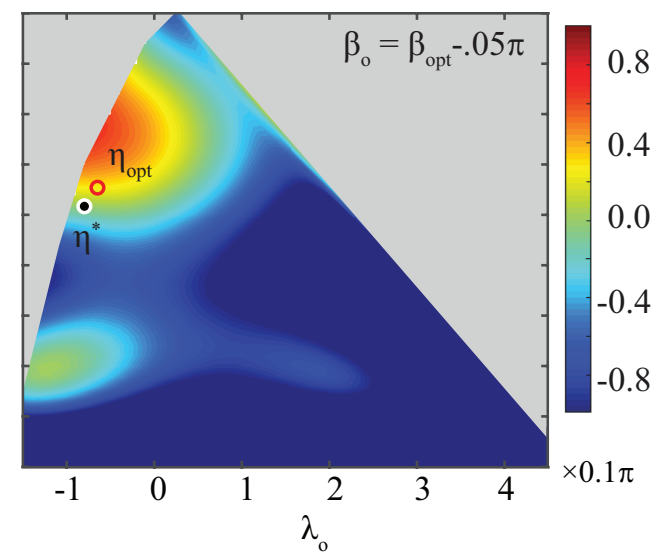

FIG. 8: "global" (A) and "local" (B) sensitivities of the transport efficiency measured from a suboptimal choice of $\beta^{*}$ as functions of $\lambda_{o}$ and $\alpha_{o}$ for $\Delta=0.05 \pi$.

ing these three parameters from experimental data. We also introduced a mathematical approach based on a sinusoidal family of time-dependent traveling waves that generate "generic" cilia-like kinematics with prescribed values of $\lambda_{o}, \alpha_{o}$ and $\beta_{o}$. To examine the performance of the various cilia kinematics, those obtained from experimental image sequences and those generated mathematically, we used two performance metrics: the net flow transported by the cilium and its efficiency.

We compared the performance of the "generic" cilialike kinematics (Fig. 3) with those reconstructed from experiments (Fig. 1). We restricted this comparison to the case of planar beating cilia due to the lack of experimental data that accurately resolve the out-of-plane cilia beating kinematics. In the planar case, one has only two design parameters: the leaning angle $\lambda_{o}$ and beating amplitude $\alpha_{o}$. The flow rates and the transport efficiencies predicted by the generic kinematics are of the same order of magnitude as those obtained from experimental data (Fig. 4). Further, the design parameters extracted from the experimental data are all located in the region of the parameter space where the transport efficiencies are highest. Together, these observations provide strong evidence that both the reduced design parameters and the generic cilia-like kinematics proposed in this work capture the salient features of cilia beating patterns.

We then presented a systematic study of the two performance metrics as a function of $\lambda_{o}, \alpha_{o}, \beta_{o}$ in the context of the generic cilia model. Our results suggest that the flow rates are positively affected by the beating amplitude $\alpha_{o}$ and the out-of-plane swinging angle $\beta_{o}$ in the sense that larger values of $\alpha_{o}$ and/or $\beta_{o}$ generate larger flow rates (Fig. 5). Indeed, both parameters amplify the asymmetry between the effective and recovery stroke, thus enhancing flow transport. Meanwhile, the transport efficiency is maximum for an optimal set of parameter values. Values $\alpha_{o}$ and $\beta_{o}$ larger than these optimal val- ues result in the cilium moving closer to the no-slip base surface. Since larger internal power is needed to move the cilium closer to the no-slip surface, such values lead to lower transport efficiency.

Finally, we examined the sensitivity of the flow rate and efficiency with respect to the design parameters. We investigated by how much the flow rate and efficiency would change as the design parameters deviate from the most efficient parameters. We called this a "global" sensitivity. We also examined a "local" sensitivity to parameter changes by computing how 'fast' the transport flow rate and efficiency would change when perturbed away from the most efficient cilium. Our results show that perturbations in the out-of-plane angle $\beta_{o}$ or beating amplitude $\alpha_{o}$ induce large sensitivities, that is, a deterioration in ciliary performance, whereas a change of the leaning angle $\lambda_{o}$ has no notable effect on the cilia performance (Fig. 7). Table I summarizes and compares the perturbation size needed in each of the design parameters to induce a $10 \%$ drop in the ciliary performance.

TABLE I: Perturbations of the design parameters $\left(\lambda_{o}, \alpha_{o}, \beta_{o}\right)$ which lead to a $10 \%$ drop in the net flow rates and transport efficiencies relative to the optimal values.

\begin{tabular}{|c|ccc|}
\hline & $\lambda_{o}$ & $\alpha_{o}$ & $\beta_{o}$ \\
\hline$\langle Q\rangle$ & - & $0.05 \pi$ & $0.03 \pi$ \\
$\eta$ & $0.12 \pi$ & $0.06 \pi$ & $0.03 \pi$ \\
\hline
\end{tabular}

Most importantly, our sensitivity analysis shows that the most efficient cilium is not most robust to perturbations in the cilia kinematics. Indeed, we presented a counterexample showing suboptimal parameters that are more robust (less sensitive) to perturbations (Fig. 8). Our results have two major implications. First, they confirm that designing for the most efficient cilium does not automatically impose any guarantees on robustness. It is our view that from the standpoint of evolutionary biol- 
ogy, robustness of design to natural or acquired variations is as important as efficiency itself. Therefore, when using genetic computational algorithms to compute ciliary design [18, 19], one has to explicitly account for robustness. Second, our methods and results provide a quantitative framework for comparing the performance of cilia from different cell type, as well as cilia from the same cell type under different operating conditions such as in healthy and diseased states. To this end, one can begin to investigate quantitatively how perturbations and/or disruptions of the ciliary apparatus, whether due to a genetic disorder or infective and acquired causes, affect the flow transport. Low and inefficient flow rates of ciliated sur- faces in mammalian organisms are directly linked to infection and disease, such as in cystic fibrosis and asthma. A quantitative map from cilia parameters to flow rates and vice versa would therefore provide an important tool for assessing cilia performance in health and disease.

\section{ACKNOWLEDGMENTS}

Computation for the work described in this paper was supported by the University of Southern California's Center for High-Performance Computing (http://hpcc.usc.edu).
[1] P. Satir and S. T. Christensen, Annual Review of Physiology 69, 377 (2007).

[2] G. R. Fulford and J. R. Blake, Journal of Theoretical Biology 121, 381 (1986).

[3] C. O'Callaghan, K. Sikand, and A. Rutman, Pediatric research 46, 704 (1999).

[4] S. H. Randell and R. C. Boucher, American journal of respiratory cell and molecular biology 35, 20 (2006).

[5] M. R. Del Bigio, Glia 14, 1 (1995).

[6] Z. Mirzadeh, Y.-G. Han, M. Soriano-Navarro, J. M. García-Verdugo, and A. Alvarez-Buylla, The Journal of Neuroscience 30, 2600 (2010).

[7] R. Lyons, E. Saridogan, and O. Djahanbakhch, Human reproduction update 12, 363 (2006).

[8] L. Wong, I. F. Miller, and D. B. Yeates, Journal of Applied Physiology 75, 458 (1993)

[9] A. Wanner, M. Salathé, and T. G. O'Riordan, American journal of respiratory and critical care medicine 154, 1868 (1996).

[10] J. R. Davenport and B. K. Yoder, American Journal of Physiology-Renal Physiology 289, F1159 (2005).

[11] S. K. Chopra, G. V. Taplin, D. H. Simmons, and D. Elam, CHEST Journal 71, 155 (1977).

[12] D. Smith, E. Gaffney, and J. Blake, Respiratory physiology \& neurobiology 163, 178 (2008).

[13] D. Smith, E. Gaffney, and J. Blake, in Proceedings of the Royal Society of London A: Mathematical, Physical and Engineering Sciences (The Royal Society, 2009) p. rspa20090018.

[14] W.-E. Li, W. Chen, Y.-F. Ma, Q.-R. Tuo, X.-J. Luo, T. Zhang, W.-B. Sai, J. Liu, J. Shen, Z.-G. Liu, et al., Pflügers Archiv-European Journal of Physiology 464, 671 (2012).

[15] Y. Ding, J. C. Nawroth, M. J. McFall-Ngai, and E. Kanso, Journal of Fluid Mechanics 743, 124 (2014).

[16] M. Fliegauf, T. Benzing, and H. Omran, Nature Reviews Molecular Cell Biology 8, 880 (2007).

[17] B. Afzelius, The Journal of pathology 204, 470 (2004).
[18] C. Eloy and E. Lauga, Physical Review Letters 109, 038101 (2012).

[19] N. Osterman and A. Vilfan, Proceedings of the National Academy of Sciences 108, 15727 (2011).

[20] H. Guo, J. Nawroth, Y. Ding, and E. Kanso, Physics of Fluids (1994-present) 26, 091901 (2014).

[21] V. A. Tucker, Comparative Biochemistry and Physiology 34, 841 (1970).

[22] Y. Katsu-Kimura, F. Nakaya, S. A. Baba, and Y. Mogami, Journal of Experimental Biology 212, 1819 (2009).

[23] M. A. Sleigh, Symposia of the Society for Experimental Biology 22, 131 (1968).

[24] G. I. Taylor, Proceedings of the Royal Society of London. Series A 209, 447 (1951).

[25] D. Eshel and C. J. Brokaw, Cell motility and the cytoskeleton 7, 160 (1987).

[26] P. Bayly, B. Lewis, P. Kemp, R. Pless, and S. Dutcher, Cytoskeleton 67, 56 (2010).

[27] P. Sartori, V. Geyer, A. Scholich, F. Jülicher, and J. Howard, arXiv preprint arXiv:1511.04270 (2015).

[28] R. Cortez, L. Fauci, and A. Medovikov, Physics of Fluids (1994-present) 17, 031504 (2005).

[29] J. Ainley, S. Durkin, R. Embid, P. Boindala, and R. Cortez, Journal of Computational Physics 227, 4600 (2008).

[30] N. Liron, Journal of Fluid Mechanics 86, 705 (1978).

[31] D. J. Smith, J. R. Blake, and E. A. Gaffney, Journal of The Royal Society Interface 5, 567 (2008).

[32] K. Machin, J. exp. Biol 35, 796 (1958).

[33] C. Brennen and H. Winet, Annual Review of Fluid Mechanics 9, 339 (1977).

[34] M. Hines and J. J. Blum, Biophysical Journal 41, 67 (1983).

[35] F. Gittes, B. Mickey, J. Nettleton, and J. Howard, The Journal of cell biology 120, 923 (1993).

[36] E. Lauga and T. R. Powers, Reports on Progress in Physics 72, 096601 (2009). 